\title{
Completeness of Determinantal Hamiltonian Flows on the Matrix Affine Poisson Space
}

\author{
MICHAEL GEKHTMAN ${ }^{1}$ and MILEN YAKIMOV ${ }^{2,3}$ \\ ${ }^{1}$ Department of Mathematics, University of Notre Dame, Notre Dame, IN 46556, USA. \\ e-mail: Michael.Gekhtman.1@nd.edu \\ ${ }^{2}$ Department of Mathematics, Louisiana State University, Baton Rouge, LA 70803, USA. \\ ${ }^{3}$ Department of Mathematics, University of California, Santa Barbara, CA 93106, USA. \\ e-mail: yakimov@math.lsu.edu
}

Received: 26 September 2008 / Revised: 1 June 2009 / Accepted: 3 June 2009

Published online: 22 September 2009 - (C) The Author(s) 2009. This article is published with open access at Springerlink.com

\begin{abstract}
The matrix affine Poisson space $\left(M_{m, n}, \pi_{m, n}\right)$ is the space of complex rectangular matrices equipped with a canonical quadratic Poisson structure which in the square case $m=n$ reduces to the standard Poisson structure on $\mathrm{GL}_{n}(\mathbb{C})$. We prove that the Hamiltonian flows of all minors are complete. As a corollary we obtain that all Kogan-Zelevinsky integrable systems on $M_{n, n}$ are complete and thus induce (analytic) Hamiltonian actions of $\mathbb{C}^{n(n-1) / 2}$ on $\left(M_{n, n}, \pi_{n, n}\right)$ (as well as on $\mathrm{GL}_{n}(\mathbb{C})$ and on $\mathrm{SL}_{n}(\mathbb{C})$ ). We define Gelfand-Zeitlin integrable systems on $\left(M_{n, n}, \pi_{n, n}\right)$ from chains of Poisson projections and prove that their flows are also complete. This is an analog for the quadratic Poisson structure $\pi_{n, n}$ of the recent result of Kostant and Wallach (Studies in Lie Theory. Progress in Mathematics, vol 243, pp 319-364. Birkhäuser, Boston, 2006) that the flows of the complexified classical Gelfand-Zeitlin integrable systems are complete.
\end{abstract}

Mathematics Subject Classification (2000). Primary 37J35; Secondary 53D17, 17 B62.

Keywords. Hamiltonian flows, matrix affine Poisson spaces, Kogan-Zelevinsky integrable systems, Gelfand-Zeitlin systems.

\section{Introduction}

The matrix affine Poisson space is the complex affine space $M_{m, n}$ consisting of rectangular matrices of size $m \times n$ equipped with the quadratic Poisson structure

$$
\pi_{m, n}=\sum_{i, k=1}^{m} \sum_{j, l=1}^{n}(\operatorname{sign}(k-i)+\operatorname{sign}(l-j)) x_{i l} x_{k j} \frac{\partial}{\partial x_{i j}} \wedge \frac{\partial}{\partial x_{k l}},
$$

where $x_{i j}$ are the standard coordinate functions on $M_{m, n}$. The geometry of this Poisson structure was studied in $[3,7,8]$, motivated by its relation to cluster algebras [6] and the ring theory of the algebra of quantum matrices [2].

The square case is closely related to the theory of Poisson Lie groups. Denote $M_{n}=M_{n, n}$ and $\pi_{n}=\pi_{n, n}$. The standard Poisson structure on $\mathrm{GL}_{n}(\mathbb{C})$, defined by 
Drinfeld, is given by

$$
\pi_{\mathrm{GL}_{n}}=\sum_{i<j}\left(\mathcal{L}_{E_{i j}} \wedge \mathcal{L}_{E_{j i}}-\mathcal{R}_{E_{i j}} \wedge \mathcal{R}_{E_{j i}}\right) .
$$

Here $\mathcal{L}$ and $\mathcal{R}$ denote the left and right invariant vector fields on $\mathrm{GL}_{n}(\mathbb{C})$, and $E_{i j}$ denote the standard elementary matrices considered as elements of $\mathfrak{g l}_{n}(\mathbb{C})$. The canonical embedding

$$
\left(\mathrm{GL}_{n}(\mathbb{C}), \pi_{\mathrm{GL}_{n}}\right) \hookrightarrow\left(M_{n}, \pi_{n}\right)
$$

is Poisson.

A canonical compactification of $\left(M_{m, n}, \pi_{m, n}\right)$ as a Poisson manifold was constructed in $[3,7]$. Define the parabolic subgroup of $\mathrm{GL}_{m+n}(\mathbb{C})$

$$
P_{n, m}=\left\{\left(\begin{array}{cc}
A & B \\
0 & C
\end{array}\right) \in \mathrm{GL}_{m+n}(\mathbb{C}) \mid A \in M_{n}, B \in M_{n, m}, C \in M_{m}\right\} .
$$

Then $\mathrm{GL}_{m+n}(\mathbb{C}) / P_{n, m}$ is diffeomorphic to the Grassmannian $\operatorname{Gr}(n, m+n)$ and $M_{m, n}$ is embedded in it as the open Schubert cell by

$$
X \in M_{m, n} \mapsto\left(\begin{array}{cc}
I_{n} & 0 \\
X & I_{m}
\end{array}\right) \cdot P_{n, m},
$$

where $I_{n}$ denotes the identity matrix of size $n \times n$. One can define the canonical Poisson structure on $\operatorname{Gr}(n, m+n)$

$$
\pi_{\mathrm{Gr}(n, m+n)}=-\sum_{1 \leq i<j \leq m+n} \chi\left(E_{i j}\right) \wedge \chi\left(E_{j i}\right),
$$

where $\chi: \mathfrak{g l}_{m+n}(\mathbb{C}) \rightarrow \operatorname{Vect}(\operatorname{Gr}(m, m+n))$ refers to the infinitesimal action induced from the left action of $\mathrm{GL}_{m+n}(\mathbb{C})$ on $\operatorname{Gr}(n, m+n)$. For a smooth manifold $M$, $\operatorname{Vect}(M)$ stands for the Lie algebra of smooth vector fields on $M$. The Poisson structure $\pi_{\mathrm{Gr}(n, m+n)}$ can be also defined as the push-forward of $\pi_{\mathrm{GL}_{m+n}}$ under the natural projection $\mathrm{GL}_{m+n}(\mathbb{C}) \rightarrow \mathrm{GL}_{m+n}(\mathbb{C}) / P_{n, m} \cong \mathrm{Gr}(n, m+n)$. The map (1.2) is not Poisson but a minor correction turns it into a Poisson map. Let us identify the symmetric group $S_{n}$ with the subgroup of $\mathrm{GL}_{n}(\mathbb{C})$ consisting of permutation matrices. Denote the longest element of $S_{n}$ by $w_{\circ}^{n}$. Then by [7, (3.11)] and [3, Proposition 3.3] the map

$$
i_{m, n}:\left(M_{m, n}, \pi_{m, n}\right) \hookrightarrow\left(\mathrm{GL}_{m+n}(\mathbb{C}) / P_{n, m}, \pi_{G L_{m+n}}\right), \quad i_{m, n}(X)=\left(\begin{array}{cc}
I_{n} & 0 \\
X w_{\circ}^{n} & I_{m}
\end{array}\right) \cdot P_{n, m}
$$

is Poisson.

Given two subsets $I \subset\{1, \ldots, m\}$ and $J \subset\{1, \ldots, n\}$ with the same number of elements, denote by $\Delta_{I, J}(X)$ the minor of $X \in M_{m, n}$ (i.e., the determinant of the submatrix of $X$ ) corresponding to rows $I$ and columns $J$. Our first result is that the Hamiltonian flows of $\Delta_{I, J}$ are complete; that is the corresponding integral 
curves are defined over all of $\mathbb{C}$. In addition, we show that the corresponding dynamics is given by

$x_{i j}(t)=p(t)+\sum_{a=1}^{N} p_{a}(t) e^{\alpha_{a} t}, \quad t \in \mathbb{C}, \quad$ for some polynomials $p(t), p_{1}(t), \ldots, p_{N}(t)$,

such that $\operatorname{deg} p(t) \leq 2|I|-1, \operatorname{deg} p_{a}(t) \leq 2|I|-2, a=1, \ldots, N$, and some $\alpha_{1}, \ldots, \alpha_{N} \in \mathbb{C}^{*}$. Here $|I|$ denotes the number of elements of $I$. The integer $N$, the polynomials $p(t), p_{a}(t)$ and the exponents $\alpha_{a}$ depend, in general, on the initial conditions and on the indices $i$ and $j$. This result is proved in Section 2.

Kogan and Zelevinsky [9] constructed completely integrable systems on symplectic leaves of the double Bruhat cells (corresponding to equal elements of the Weyl group) of any complex reductive Lie group, equipped with the standard Poisson structure. Their Hamiltonians are either twisted generalized minors or generalized minors. If we restrict ourselves to $\mathrm{GL}_{n}(\mathbb{C})$, this construction produces families of $(n-1)(n-2) / 2$ functionally independent Hamiltonians on $M_{n}$ (given by minors) parametrized by reduced expressions of the longest element $w_{\circ}^{n} \in S_{n}$. Our result implies that, in the $A_{n-1}$ case, the flows of all Kogan-Zelevinsky integrable systems are complete, even when extended to $M_{n}$. In particular each of these systems provides a Hamiltonian action of $\mathbb{C}^{(n-1)(n-2) / 2}$ on $\left(M_{n}, \pi_{n}\right)$ (and on $\left(\mathrm{GL}_{n}(\mathbb{C}), \pi_{\mathrm{GL}_{n}}\right)$ and on $\left.\left(\mathrm{SL}_{n}(\mathbb{C}), \pi_{\mathrm{GL}_{n}} \mid \mathrm{SL}_{n}\right)\right)$. The action is analytic but not algebraic. This is carried out in Section 3. One should note here that, although the left and right actions of the standard maximal torus of $\mathrm{GL}_{n}(\mathbb{C})$ preserve $\pi_{\mathrm{GL}_{n}}$, they are not Hamiltonian. In addition, they provide Abelian symmetry groups which are much smaller than the ones constructed in this paper (of dimension $(n-1)(n-2) / 2$ ).

Kostant and Wallach [10] have recently shown that all flows of the complexified Gelfand-Zeitlin integrable systems are complete everywhere on $\mathfrak{g l} l_{n}^{*}(\mathbb{C})$. (This is not true for the flows of the classical system on $\mathfrak{u}_{n}$.) This paper grew out of our attempt to understand whether a similar fact is true for a Gelfand-Zeitlin integrable system for the quadratic Poisson structure $\pi_{n}$ on $M_{n}$. We construct a GelfandZeitlin type integrable system on $\left(M_{n}, \pi_{n}\right)$ as follows. Any projection from $\left(M_{n}, \pi_{n}\right)$ to $\left(M_{k}, \pi_{k}\right)$ sending an $n \times n$ matrix to (any) given $k \times k$ submatrix is Poisson for all $k<n$. We get the chain of Poisson maps

$$
\left(M_{n}, \pi_{n}\right) \rightarrow\left(M_{n-1}, \pi_{n-1}\right) \rightarrow \cdots \rightarrow\left(M_{1}, \pi_{1}\right)
$$

by sending a $k \times k$ matrix to its principal $(k-1) \times(k-1)$ submatrix for all $k=$ $n, n-1, \ldots, 2$. The field of rational functions on any $M_{k}$ is a Poisson field. We determine its Poisson center $Z\left(\mathbb{C}\left(M_{k}\right)\right)$ (i.e., its center with respect to the Poisson bracket) using a result of Kogan and Zelevinsky [9], see Proposition 4.2 for details. Pulling back all Poisson centers to $\mathbb{C}\left(M_{n}\right)$ produces the Poisson commutative subfield of $\mathbb{C}\left(M_{n}\right)$ which is a pure transcendental extension of $\mathbb{C}$ by

$$
\Delta_{l ; k} / \Delta_{l ; l-k}, \quad 1 \leq k<l \leq n, \Delta_{l}, \quad 1 \leq l \leq n .
$$


We call the set of Hamiltonians (1.3) the Gelfand-Zeitlin integrable system for the quadratic Poisson bracket $\pi_{n}$. Those Hamiltonians indeed define an integrable system on any symplectic leaf of the open double Bruhat cell in $\mathrm{GL}_{n}(\mathbb{C})$. Every such leaf has dimension $n(n-1) / 2$.

Finally we prove that the flow of each of the Hamiltonians (1.3) is complete in the following sense (see Theorem 4.5). Denote by $D_{n}$ the subvariety of $M_{n}$ which is the union of the zero level sets of the denominators of the Hamiltonians in (1.3). Fix $h$ to be one of the Hamiltonians (1.3) and $X_{0} \in M_{n} \backslash D_{n}$. We prove that there exists a curve $\gamma: \mathbb{C} \rightarrow M_{n}$ whose entry functions are given by

$$
\sum_{p=1}^{N}\left(c_{i j}^{p}+d_{i j}^{p} t\right) e^{\alpha_{i j}^{p} t}+\left(b_{i j}+c_{i j} t+d_{i j} t^{2}\right)
$$

for some integer $N$ and $b_{i j}, c^{i j}, d^{i j}, c_{i j}^{p}, d_{i j}^{p} \in \mathbb{C}, \alpha_{i j}^{p} \in \mathbb{C}^{*}$ depending on $X_{0}$ with the following properties:

1. $\gamma^{-1}\left(D_{n}\right)$ is a discrete subset of $\mathbb{C}$ and

2. $\gamma: \mathbb{C} \backslash \gamma^{-1}\left(D_{n}\right) \rightarrow M_{n}$ is the integral curve of the Hamiltonian $h$ such that $\gamma(0)=X_{0}$.

\section{Completeness of Determinantal Hamiltonian Flows}

Let

$$
I=\left\{i_{1}<\cdots<i_{r}\right\} \subset\{1, \ldots, m\}, \quad J=\left\{j_{1}<\cdots<j_{r}\right\} \subset\{1, \ldots, n\} .
$$

For $k \in\{1, \ldots, n\}$ set

$$
I\left(i_{p} \rightarrow k\right)=\left(I \backslash\left\{i_{p}\right\}\right) \cup\{k\} .
$$

Recall the Poisson brackets [7]

$$
\left\{x_{k l}, \Delta_{I, J}\right\}=\sum_{q=1}^{r} \operatorname{sign}\left(i_{q}-k\right) x_{i_{q} l} \Delta_{I\left(i_{q} \rightarrow k\right), J}+\sum_{q=1}^{r} \operatorname{sign}\left(j_{q}-l\right) x_{k j_{q}} \Delta_{I, J\left(j_{q} \rightarrow l\right)},
$$

for all $k=1, \ldots, m$ and $l=1, \ldots, n$ (cf. the proof of [7, Lemma 3.2]). Following [7], we say that $\operatorname{sign}(I-k)$ is defined if $k \in I, k<i_{1}$, or $k>i_{r}$ in which cases it is equal to 0,1 , or -1 , respectively. We recall the following lemma for the convenience of the reader.

LEMMA 2.1 [7, Lemma 3.2]. If, in the above notation, $\operatorname{sign}(I-k)$ and $\operatorname{sign}(J-l)$ are defined and

$$
|\operatorname{sign}(I-k)+\operatorname{sign}(J-l)| \leq 1,
$$

then

$$
\left\{x_{k l}, \Delta_{I, J}\right\}=(\operatorname{sign}(I-k)+\operatorname{sign}(J-l)) x_{k l} \Delta_{I, J} .
$$


The main result of this section is:

THEOREM 2.2. The Hamiltonian flow of any minor $\Delta_{I, J}$ on $\left(M_{m, n}, \pi_{m, n}\right)$ is complete over $\mathbb{C}$. In addition, each matrix entry evolves quasi-exponentially according to this flow:

$$
x_{k l}(t)=p(t)+\sum_{a=1}^{N} p_{a}(t) e^{\alpha_{a} t}, \quad t \in \mathbb{C}, \quad \text { for some polynomials } p(t), p_{1}(t), \ldots, p_{N}(t),
$$

such that $\operatorname{deg} p(t) \leq 2 r-1, \operatorname{deg} p_{a}(t) \leq 2 r-2, a=1, \ldots, N$, and some $\alpha_{1}, \ldots, \alpha_{N} \in$ $\mathbb{C}^{*}$. The integer $N$, the polynomials $p(t), p_{a}(t)$, and the exponents $\alpha_{a}$ depend on the initial condition, $k$ and $l$.

Proof. Assume that $I$ and $J$ are given by (2.1). We will consider several cases for $k$ and $l$.

Case 1. $\left(k>i_{r}, l<j_{1}\right)$ or $\left(k<i_{1}, l>j_{r}\right)$ or $(k \in I, l \in J)$. In this case, $\operatorname{sign}(I-k)$ and $\operatorname{sign}(J-l)$ are defined, and $\operatorname{sign}(I-k)+\operatorname{sign}(J-l)=0$. According to Lemma 2.1

$$
x_{k l}^{\prime}(t)=\left\{x_{k l}, \Delta_{I, J}\right\}(X(t))=0,
$$

where $X(t)$ denotes the matrix with entries $x_{i j}(t), i=1, \ldots, m, j=1, \ldots, n$. Thus $x_{k l}(t)=x_{k l}(0)$ for all $t \in \mathbb{C}$.

Case 2. $(k \notin I, l \in J)$. The case $(k \in I, l \notin J)$ is treated analogously. In this case (2.2) implies

$$
x_{k l}^{\prime}(t)=\sum_{q=1}^{r} \operatorname{sign}\left(i_{q}-k\right) x_{i_{q} l}(t) \Delta_{I\left(i_{q} \rightarrow k\right), J}(X(t)),
$$

where $X(t)$ is the $m \times n$ matrix as in Case 1. Taking into account Case 1, we obtain that

$$
x_{k l}^{\prime}(t)=c_{k l j_{1}} x_{k j_{1}}(t)+\cdots+c_{k l j_{r}} x_{k j_{r}}(t)
$$

for some constants $c_{k j_{a} j_{b}}(k \notin I, a, b=1, \ldots, r)$ which depend on the initial condition. Thus the vectors

$$
\vec{x}_{k}(t)=\left(x_{k j_{1}}(t), \ldots, x_{k j_{r}}(t)\right)^{T}, \quad k \notin I
$$

satisfy

$$
\vec{x}_{k}^{\prime}(t)=C_{k} \vec{x}_{k}(t)
$$

for the constant matrices $C_{k}=\left(c_{k j_{a} j_{b}}\right)_{a, b=1}^{r}$. Therefore, in this case, the entries $x_{k l}(t)$ evolve according to (2.3) with $\operatorname{deg} p(t)+\operatorname{deg} p_{1}(t)+\cdots+\operatorname{deg} p_{N}(t) \leq r-N-1$. 
Case 3. $(k \notin I, l \notin J)$. Equation (2.2) and Cases 1 and 2 imply

$$
x_{k l}^{\prime}(t)=q_{0}(t)+\sum_{a=1}^{N} q_{a}(t) e^{\alpha_{a} t}, \quad t \in \mathbb{C},
$$

for some polynomials $q_{0}(t), \ldots, q_{N}(t)$ such that $\operatorname{deg} q_{a}(t) \leq 2 r-2, a=0, \ldots, N$ and some $\alpha_{1}, \ldots, \alpha_{N} \in \mathbb{C}^{*}$. The statement of the theorem now follows from this. Note that some of the cases in 1 are included in Case 3, but the dynamics in 1 is simpler.

\section{Completeness of the Kogan-Zelevinsky Integrable Systems on $\left(M_{n}, \pi_{n}\right)$ and Hamiltonian Group Actions}

In [9], Kogan and Zelevinsky constructed completely integrable systems on certain symplectic leaves of a complex simple group $G$ equipped with the standard Poisson structure (the leaves in all double Bruhat cells of the type $G^{u, u}$ for some element $u$ of the corresponding Weyl group). We restrict our attention to $\mathrm{GL}_{n}$ and the leaves in the open double Bruhat cell $\mathrm{GL}_{n}^{w_{\circ}^{n}, w_{\circ}^{n}}=B_{+} w_{\circ}^{n} B_{+} \cap B_{-} w_{\circ}^{n} B_{-}$(recall that $w_{\circ}^{n}$ denotes the longest element of $\left.S_{n}\right)$. Since $B_{+} w_{\circ}^{n} B_{+} \cap B_{-} w_{\circ}^{n} B_{-}$is Zariski open in $M_{n}$ and the corresponding Hamiltonians are regular functions on $M_{n}$, the KoganZelevinsky integrable systems for this case provide sets of $n(n-1) / 2$ functionally independent commuting Hamiltonians on $\left(M_{n}, \pi_{n}\right)$.

Denote by $s_{1}, \ldots, s_{n-1}$ the simple reflections in $S_{n}$. For two integers $k \leq l$ set

$$
[k, l]=\{k, k+1, \ldots, l\} .
$$

Fix a reduced expression for the longest element of $S_{n}$ :

$$
w_{\circ}^{n}=s_{j_{1}} \cdots s_{j_{n(n-1) / 2}} .
$$

Following [9], for $k=1, \ldots, n(n-1) / 2$ set

$$
v_{k}=s_{j_{1}} \cdots s_{j_{k-1}}, \quad u_{k}=\left(w_{\circ}^{n}\right)^{-1} v_{k}=s_{j_{n(n-1) / 2}} \cdots s_{j_{k}}
$$

and define the Hamiltonians

$$
H_{k}=\Delta_{u_{k}[1, k], v_{k}[1, k]} \in \mathbb{C}\left[M_{n}\right], \quad k=1, \ldots, n(n-1) / 2 .
$$

THEOREM 3.1 [9]. For any reduced expression (3.2) of the longest element of $S_{n}$, the Hamiltonians $H_{1}, \ldots, H_{n(n-1) / 2}$ Poisson commute with respect to $\pi_{n}$.

Theorem 2.2 has the following immediate corollary.

COROLLARY 3.2. For any reduced expression (3.2) of the longest element of $S_{n}$ the flows of the Kogan-Zelevinsky Hamiltonians (3.3) are complete on $\left(M_{n}, \pi_{n}\right)$. As 
a consequence, from each such reduced expression one obtains an analytic Hamiltonian action of $\mathbb{C}^{n(n-1) / 2}$ on $\left(M_{n}, \pi_{n}\right)$.

As was pointed out in the introduction, the left and right actions of the standard maximal torus of $\mathrm{GL}_{n}(\mathbb{C})$ are not Hamiltonian and provide much smaller Abelian symmetry groups of $\left(M_{n}, \pi_{n}\right)$.

\section{Gelfand-Zeitlin Integrable Systems on $\left(M_{n}, \pi_{n}\right)$}

The field of rational functions $\mathbb{C}(P)$ of an irreducible affine complex Poisson variety $(P, \pi)$ is naturally a Poisson field. Its center with respect to the Poisson bracket will be denoted by $Z(\mathbb{C}(P))$ and will be called the Poisson center of $\mathbb{C}(P)$.

\subsection{POISSON MAPS BETWEEN DIFFERENT $\left(M_{m, n}, \pi_{m, n}\right)$}

For two subsets $I \subset\{1, \ldots, m\}$ and $J \subset\{1, \ldots, n\}$ we have the natural projection and embedding maps:

$$
\phi_{I, J}^{m, n}: M_{m, n} \rightarrow M_{|I|,|J|}, \quad i_{I, J}^{m, n}: M_{|I|,|J|} \hookrightarrow M_{m, n},
$$

where $|I|$ and $|J|$ denote the number of elements of $I$ and $J$. Given $X \in M_{m, n}$, $\phi_{I, J}^{m, n}(X)$ denotes the submatrix of $X$ corresponding to rows in $I$ and columns in $J$. For $X \in M_{|I|,|J|}, i_{I, J}^{m, n}(X) \in M_{m, n}$ is the matrix whose $I, J$ th submatrix is $X$ and all other entries are equal to 0 .

One immediately checks that:

LEMMA 4.1. For all subsets $I \subset\{1, \ldots, m\}, J \subset\{1, \ldots, n\}$ the maps

$$
\phi_{I, J}^{m, n}: M_{m, n} \rightarrow M_{|I|,|J|} \text { and } i_{I, J}^{m, n}: M_{|I|,|J|} \rightarrow M_{m, n}
$$

are morphisms of affine Poisson spaces.

Note that if one identifies $M_{n}$ with $\mathfrak{g l}_{n}^{*}(\mathbb{C})$, then for the Kirillov-Kostant Poisson structure the morphisms $\phi_{I, J}^{n, n}$ are Poisson only if $I=J$, while none of the $i_{I, J}^{n, n}$ is Poisson.

\subsection{THE POISSON CENTER OF $\mathbb{C}\left(M_{n}\right)$}

We will construct Poisson commutative subfields of $\mathbb{C}\left(M_{n}\right)$ by pulling back Poisson centers under a chain of projection maps. For this purpose, we will need a description of the Poisson centers $Z\left(\mathbb{C}\left(M_{n}\right)\right)$.

For $1 \leq k<l \leq n$ define $\Delta_{l ; k}, \Delta_{l ; k}^{\prime} \in \mathbb{C}\left[M_{n}\right]$ by

$$
\Delta_{l ; k}(X)=\Delta_{[1, k],[l-k+1, l]}(X) \text { quadand } \quad \Delta_{l ; k}^{\prime}(X)=\Delta_{[l-k+1, l],[1, k]}(X), \quad X \in M_{n} .
$$


In other words $\Delta_{l ; k}(X)$ and $\Delta_{l ; k}^{\prime}(X)$ are the upper-right $k \times k$ minor and the lower-left $k \times k$ minor of $\phi_{[1, l],[1, l]}^{n, n}(X)$.

For $1 \leq l \leq n$, define also $\Delta_{l} \in \mathbb{C}\left[M_{n}\right]$ by

$$
\Delta_{l}(X)=\Delta_{[1, l],[1, l]}(X)=\operatorname{det}\left(\phi_{[1, l],[1, l]}(X)\right), \quad X \in M_{n} .
$$

The following proposition describes the Poisson centers $Z\left(\mathbb{C}\left(M_{n}\right)\right)$.

PROPOSITION 4.2. The (Poisson) center of the Poisson field $\mathbb{C}\left(M_{n}\right)$ is

$$
\mathbb{C}\left(\Delta_{n ; 1} / \Delta_{n ; n-1}^{\prime}, \ldots, \Delta_{n ; n-1} / \Delta_{n ; 1}^{\prime}, \Delta_{n}\right) .
$$

This proposition will be obtained as an easy consequence of the following theorem of Kogan and Zelevinsky (which is a special case of their result [9, Theorem 2.3] describing the symplectic leaves of the standard Poisson structure on any complex simple Lie group, see [9, Example 2.10]).

THEOREM 4.3. (Kogan-Zelevinsky) The symplectic leaves of the Zariski open subset $\mathrm{GL}_{n}^{w_{\circ}^{m}, w_{\circ}^{n}}$ of $M_{n}$ are

$$
\begin{array}{r}
\left\{X \in \mathrm{GL}_{n}^{w_{\circ}^{m}, w_{\circ}^{n}} \mid \Delta_{n ; 1}(X) / \Delta_{n ; n-1}^{\prime}(X)=\text { const }, \ldots,\right. \\
\left.\Delta_{n ; n-1}(X) / \Delta_{n ; 1}^{\prime}(X)=\text { const, } \Delta_{n}(X)=\text { const }\right\} .
\end{array}
$$

It is clear that Theorem 4.3 implies the inclusion

$$
Z\left(M_{n}\right) \supseteq \mathbb{C}\left(\Delta_{n ; 1} / \Delta_{n ; n-1}^{\prime}, \ldots, \Delta_{n ; n-1} / \Delta_{n ; 1}^{\prime}, \Delta_{n}\right) .
$$

The major point of Proposition 4.2 is the equality of the above fields.

Proof of Proposition 4.2. Clearly $Z\left(\mathbb{C}\left(M_{n}\right)\right)=Z\left(\mathbb{C}\left(\mathrm{GL}_{n}^{w_{\circ}^{n}, w_{\circ}^{n}}\right)\right)$. Consider the map

$$
\psi: \mathrm{GL}^{w_{\circ}^{n}, w_{\circ}^{n}} \rightarrow\left(\mathbb{C}^{*}\right)^{\times n}, \quad \psi=\left(\Delta_{n ; 1} / \Delta_{n ; n-1}^{\prime}, \ldots, \Delta_{n ; n-1} / \Delta_{n ; 1}^{\prime}, \Delta_{n}\right) .
$$

Any $f \in Z\left(\mathbb{C}\left(\mathrm{GL}^{w_{\circ}^{m}, w_{\circ}^{n}}\right)\right)$ has to be constant along the generic symplectic leaves of $\left(\mathrm{GL}^{w_{\circ}^{m}, w_{\circ}^{n}}, \pi_{n}\right)$. Because of the Kogan-Zelevinsky theorem such an $f$ has to be constant along the generic fibers of $\psi$. Now the theorem follows from the fact that

$$
\mathbb{C}\left(M_{n}\right)=\mathbb{C}\left(\Delta_{n ; k} / \Delta_{n ; n-k}^{\prime}, 1 \leq k<n, \Delta_{n}, x_{i j}, 1<i \leq n, 1 \leq j \leq n\right)
$$

(in particular $\mathbb{C}\left(M_{n}\right) / \psi^{*} \mathbb{C}\left(\left(\mathbb{C}^{*}\right)^{\times n}\right)$ is a purely transcendental extension). This is proved by checking that

$$
\Delta_{n ; k} / \Delta_{n ; n-k}^{\prime}=\sum_{d=1}^{k} Q_{k d} x_{1, n+1-d}, \quad k=1, \ldots, n-1, \quad \Delta_{n}=\sum_{d=1}^{n} Q_{n d} x_{1, n+1-d}
$$


for some

$$
Q_{k d} \in \mathbb{C}\left(x_{i j}, 1<i \leq n, 1 \leq j \leq n\right), \quad 1 \leq d \leq k \leq n, \quad Q_{k k} \neq 0 .
$$

\subsection{A GELFAND-ZEITLIN TYPE INTEGRABLE SYSTEM}

For $k=2, \ldots, n$ set

$$
\phi_{k}=\phi_{[1, k-1],[1, k-1]}^{k, k}:\left(M_{k}, \pi_{k}\right) \rightarrow\left(M_{k-1}, \pi_{k-1}\right) .
$$

We have the chain of Poisson maps

$$
\left(M_{n}, \pi_{n}\right) \stackrel{\phi_{n}}{\longrightarrow}\left(M_{n-1}, \pi_{n-1}\right) \stackrel{\phi_{n-1}}{\longrightarrow} \cdots \stackrel{\phi_{2}}{\longrightarrow}\left(M_{1}, \pi_{1}\right),
$$

cf. Lemma 4.1. Following the Gelfand-Zeitlin procedure we induce a Poisson commutative subfield of $\mathbb{C}\left(M_{n}\right)$ by pulling back the Poisson centers of all fields $\mathbb{C}\left(M_{k}\right)$ and considering the subfield of $\mathbb{C}\left(M_{n}\right)$ generated by them. More precisely, set

$$
\phi_{n ; k}=\phi_{n} \cdots \phi_{k+1}:\left(M_{n}, \pi_{n}\right) \rightarrow\left(M_{k}, \pi_{k}\right)
$$

and consider the Poisson commutative subfield of $\mathbb{C}\left(M_{n}\right)$ generated by $\phi_{n ; k}^{*}\left(\mathbb{C}\left(M_{k}\right)\right)$ for all $k=1, \ldots n$. Because of Proposition 4.2 this is the field

$$
\mathbb{C}\left(\Delta_{l ; k} / \Delta_{l ; l-k}^{\prime}, 1 \leq k<l \leq n, \Delta_{l}, 1 \leq l \leq n\right) .
$$

This proves the Poisson commutativity of the set of rational Hamiltonians

$$
\Delta_{l ; k} / \Delta_{l ; l-k}^{\prime}, 1 \leq k<l \leq n, \Delta_{l}, 1 \leq l \leq n
$$

on $\left(M_{n}, \pi_{n}\right)$. We call this set the Gelfand-Zeitlin integrable system on the matrix affine Poisson space $\left(M_{n}, \pi_{n}\right)$. Let us note that the commutativity of the Hamiltonians can be also deduced from [7, Proposition 3.4].

The set of Hamiltonians (4.4) induces integrable systems on the leaves of the Zariski open subset $\mathrm{GL}_{n}^{w_{0}^{n}, w_{\circ}^{n}}$ as follows. Among those Hamiltonians, $n$ are Casimirs for $\left(\mathrm{GL}_{n}^{w_{0}^{n}, w_{0}^{n}}, \pi_{n}\right)$ and restrict to constants, cf. Theorem 4.3. The remaining $n(n-1) / 2$ are functionally independent on every symplectic leaf of $\mathrm{GL}_{n}^{w_{0}^{n}, w_{o}^{n}}$ because iterating (4.2) gives

$$
\mathbb{C}\left(M_{n}\right)=\mathbb{C}\left(\Delta_{l ; k} / \Delta_{l ; l-k}^{\prime}, 1 \leq k<l \leq n, \Delta_{l}, 1 \leq l \leq n, x_{i j}, 1 \leq j<i \leq n\right) .
$$

Since all leaves of $\mathrm{GL}_{n}^{w_{0}^{n}, w_{\circ}^{n}}$ have dimension $n(n-1)$, the Hamiltonians (4.4) induce an integrable system on each leaf.

The integrable systems (4.4) are related to the Gelfand-Zeitlin integrable systems defined by Flaschka and Ratiu [4,5] in the sense that they are produced by similar procedures but live on dual Poisson Lie groups. The one of Flaschka 
and Ratiu is on the dual Poisson-Lie group $U_{n}^{*}$ of $U_{n}$ (and thus its complexification is a Hamiltonian system on the dual Poisson Lie group of $\mathrm{GL}_{n}(\mathbb{C})$ ). The Hamiltonians (4.4) define dynamics on $M_{n}$ and on its Poisson submanifold $\mathrm{GL}_{n}(\mathbb{C})$. It was conjectured by Flaschka and Ratiu [4,5], and proved by Alekseev and Meinrenken [1] that the Gelfand-Zeitlin integrable systems on $U_{n}^{*}$ are globally isomorphic to the classical ones. At the same time the systems (4.4) are not isomorphic to Hamiltonian systems for linear Poisson structures since the standard Poisson structure on $\mathrm{GL}_{n}(\mathbb{C})$ cannot be globally linearized.

Remark 4.4. Instead of the chain of maps (4.3) one can consider any chain of projections $\phi_{I, J}^{k, k}$ where $|I|=|J|$. Clearly there are $n !^{2}$ different chains of this type. For each of them one can obtain a set of $n(n-1) / 2$ functionally independent commuting Hamiltonians on $\left(M_{n}, \pi_{n}\right)$. The resulting integrable systems are different since the right and left actions of $S_{n}$ on $M_{n}$ (permuting rows and columns) do not preserve the Poisson structure $\pi_{n}$.

\subsection{COMPLETENESS OF THE FLOWS OF THE GELFAND-ZEITLIN INTEGRABLE} SYSTEM FOR $\left(M_{n}, \pi_{n}\right)$

Denote the singular locus of the Hamiltonians (4.4) by

$$
D_{n}=\left\{X \in M_{n} \mid \Delta_{l ; k}^{\prime}(X)=0 \text { for some } 1 \leq k<l \leq n\right\} .
$$

We prove that the flows of the Hamiltonians (4.4) are complete in the following sense.

THEOREM 4.5. Let $h$ be one of the Hamiltonians (4.4) and $X_{0} \in M_{n} \backslash D_{n}$.

There exists a curve $\gamma: \mathbb{C} \rightarrow M_{n}$ whose entry functions are given by

$$
\sum_{p=1}^{N}\left(c_{i j}^{p}+d_{i j}^{p} t\right) e^{\alpha_{i j}^{p} t}+\left(b_{i j}+c_{i j} t+d_{i j} t^{2}\right)
$$

for some integer $N$ and $b_{i j}, c_{i j}, d_{i j}, c_{i j}^{p}, d_{i j}^{p} \in \mathbb{C}, \alpha_{i j}^{p} \in \mathbb{C}^{*}$ depending on $X_{0}$ with the following properties:

1. $\gamma^{-1}\left(D_{n}\right)$ is a discrete subset of $\mathbb{C}$ and

2. $\gamma: \mathbb{C} \backslash \gamma^{-1}\left(D_{n}\right) \rightarrow M_{n}$ is the integral curve of the Hamiltonian $h$ such that $\gamma(0)=X_{0}$.

Proof. We will prove the statement for the case $h=\Delta_{l ; k} / \Delta_{l ; l-k}^{\prime}$ for some $1 \leq k<$ $l \leq n$. The case of $h=\Delta_{l}$ is simpler and is left to the reader.

1. Let $i, j \in[1, l]$. Then

$$
\left\{x_{i j}, \Delta_{l ; k} / \Delta_{l ; l-k}^{\prime}\right\}=0
$$


because the map $\phi_{[1, k],[1, k]}^{n, n}$ is Poisson and we define

$$
\gamma_{i j}(t)=\left(X_{0}\right)_{i j}=\text { const }
$$

for those $i, j$ 's.

2. Let $i \in[1, k], j \in[l+1, n]$ or $i \in[l+1, n], j \in[1, l-k]$. We set $\epsilon=-1$ and 1 , respectively. From Lemma 2.1 one gets

$$
\left\{x_{i j}, \Delta_{l ; k} / \Delta_{l ; l-k}^{\prime}\right\}=\epsilon x_{i j} \Delta_{l ; k} / \Delta_{l ; l-k}^{\prime} .
$$

Define

$$
\gamma_{i j}(t)=\left(X_{0}\right)_{i j} e^{\epsilon \Delta_{l ; k}\left(X_{0}\right) t / \Delta_{l ; l-k}^{\prime}\left(X_{0}\right)} .
$$

For those values of $i$ and $j$, this is the evolution of the matrix entries of the integral curve of $h$ through $X_{0}$ outside the singular locus $D_{n}$.

3. Let $i \in[k+1, l], j \in[l+1, n]$. Using (2.2) and Lemma 2.1 we get

$$
\begin{aligned}
\left\{x_{i j}, \Delta_{l ; k} / \Delta_{l ; l-k}^{\prime}\right\}= & -\left(\sum_{q=1}^{k} x_{q j} \Delta_{[1, k](q \rightarrow i),[l-k+1, l]}+\right. \\
& \left.+\sum_{q=l-k+1}^{l} x_{i q} \Delta_{[1, k],[l-k+1, l](q \rightarrow j)}-\Delta_{l ; k}\right) / \Delta_{l ; l-k}^{\prime} .
\end{aligned}
$$

Replacing $x_{a b}$ by $\gamma_{a b}(t)$ from (4.6) and (4.7) in the right-hand side of (4.8), we see that outside $D_{n}$ the matrix entries of the integral curve of $h$ through $X_{0}$ satisfy

$$
x_{i j}^{\prime}(t)=\sum_{p=1}^{N} c_{i j}^{p} e^{\alpha_{i j}^{p} t}+d_{i j}
$$

and thus

$$
x_{i j}(t)=\sum_{p=1}^{N} c_{i j}^{p} e^{\alpha_{i j}^{p} t}+\left(c_{i j}+d_{i j} t\right)
$$

for some integer $N$ and $c^{i j}, d^{i j}, c_{i j}^{p} \in \mathbb{C}, \alpha_{i j}^{p} \in \mathbb{C}^{*}$. For $i \in[k+1, l], j \in[l+1, n]$ define $\gamma_{i j}(t)$ by the right-hand side of (4.10).

4. Let $i \in[l+1, n], j \in[l-k+1, l]$. In this case one computes the Poisson bracket $\left\{x_{i j}, \Delta_{l ; k} / \Delta_{l ; l-k}^{\prime}\right\}$ as in (4.8) and proves that it is given by a formula of the type (4.9) where again $c^{i j}, d^{i j}, c_{i j}^{p} \in \mathbb{C}, \alpha_{i j}^{p} \in \mathbb{C}^{*}$ depend on $X_{0}$. For $i \in[l+1, n]$, $j \in[l-k+1, l]$ we define $\gamma_{i j}(t)$ by the right-hand side of the latter formula. That formula also gives the evolution of the matrix entries of the integral curve of $h$ through $X_{0}$ outside the singular locus $D_{n}$. 
5. Let $i, j \in[l+1, n]$. Using (2.2) one obtains

$$
\begin{aligned}
\left\{x_{i j}, \Delta_{l ; k} / \Delta_{l ; l-k}^{\prime}\right\}= & -\left(\sum_{q=1}^{k} x_{q j} \Delta_{[1, k](q \rightarrow i),[l-k+1, l]}+\right. \\
& \left.+\sum_{q=l-k+1}^{l} x_{i q} \Delta_{[1, k],[l-k+1, l](q \rightarrow j)}\right) / \Delta_{l ; l-k}^{\prime}+ \\
& +\Delta_{l ; k}\left(\sum_{q=1}^{k} x_{q j} \Delta_{[k+1, l](q \rightarrow i),[1, l-k]}+\right. \\
& \left.+\sum_{q=l-k+1}^{l} x_{i q} \Delta_{[k+1, l],[1, l-k](q \rightarrow j)}\right) /\left(\Delta_{l ; l-k}^{\prime}\right)^{2}
\end{aligned}
$$

We replace $x_{a b}$ by $\gamma_{a b}(t)$ from the formulas of cases 1-4 in the right-hand side of (4.11), and we see that outside $D_{n}$ the matrix entries of the integral curve of $h$ through $X_{0}$ satisfy

$$
x_{i j}^{\prime}(t)=\sum_{p=0}^{N}\left(c_{i j}^{p}+d_{i j}^{p} t\right) e^{a_{i j}^{p} t}
$$

for some $c_{i j}^{p}, \alpha_{i j}^{p} \in \mathbb{C}$. We will assume that $\alpha_{i j}^{0}=0$ and set $c_{i j}^{0}=d_{i j}^{0}=0$ if polynomial terms are not present. Thus, outside $D_{n}$ the integral curve of $h$ through $X_{0}$ is integrated to

$$
x_{i j}(t)=\sum_{p=1}^{N}\left(c_{i j}^{p}+d_{i j}^{p} t\right) e^{a_{i j}^{p} t}+\left(b_{i j}+c_{i j} t+d_{i j} t^{2}\right)
$$

for some $b_{i j}, c_{i j}, d_{i j}, c_{i j}^{p}, d_{i j}^{p} \in \mathbb{C}, \alpha_{i j}^{p} \in \mathbb{C}^{*}$. For $i, j \in[l+1, n]$ we define $\gamma_{i j}(t)$ by the right-hand side of (4.13).

In cases $1-5$, the integral curve of $h$ is an analytic curve $\gamma: \mathbb{C} \rightarrow M_{n}$ with the property (4.5). If $h$ is any of the Hamiltonians (4.4) and $\Delta$ is its denominator, then $\Delta(\gamma(t))$ is an entire function which does not vanish identically since $\Delta(\gamma(0))=$ $\Delta\left(X_{0}\right) \neq 0$. Therefore

$$
\gamma^{-1}\left(\left\{X \in M_{n} \mid \Delta(X)=0\right\}\right)
$$

is a discrete subset of $\mathbb{C}$. Thus $\gamma^{-1}\left(D_{n}\right)$ is a discrete subset of $\mathbb{C}$ since it is the union of all sets (4.14) where $h$ runs over the Hamiltonians (4.4). Finally, outside $\gamma^{-1}\left(D_{n}\right), \gamma(t)$ is the integral curve of the Hamiltonian $h$ through $X_{0}$ because of the way the matrix entries of $\gamma(t)$ were constructed. 


\section{Acknowledgements}

We would like to thank Yvette Kosmann-Schwarzbach and the referees for many valuable comments which helped us to improve the paper a lot. M.Y. would like to thank the organizers of the conference Poisson 2008 for the invitation to participate in this very stimulating conference. The research of M. G. was partially supported by NSF grant DMS-0801204. The research of M.Y. was partially supported by NSF grant DMS-0701107 and an Alfred P. Sloan Research Fellowship.

Open Access This article is distributed under the terms of the Creative Commons Attribution Noncommercial License which permits any noncommercial use, distribution, and reproduction in any medium, provided the original author(s) and source are credited.

\section{References}

1. Alekseev, A., Meinrenken, E.: Ginzburg-Weinstein via Gelfand-Zeitlin. J. Differ. Geom. 76, 1-34 (2007)

2. Brown, K.A., Goodearl, K.R.: Lectures on Algebraic Quantum Groups, Advanced Courses in Mathematics. CRM Barcelona, Birkhäuser, Basel (2002)

3. Brown, K.A., Goodearl, K.R., Yakimov, M.: Poisson structures of affine spaces and flag varieties. I. Matrix affine Poisson space. Adv. Math. 206, 567-629 (2006)

4. Flaschka, H., Ratiu, T.: A convexity theorem for Poisson actions of compact Lie groups, IHES preprint 1995. http://preprints.cern.ch

5. Flaschka, H., Ratiu, T.: A Morse-theoretic proof of Poisson Lie convexity. In: Integrable Systems and Foliations/Feuilletages et systèmes intégrables (Montpellier, 1995). Progress in Mathematics, vol. 145, pp. 49-71. Birkhäuser, Boston (1997)

6. Fomin, S., Zelevinsky, A.: Cluster algebras. I. Foundations. J. Am. Math. Soc. 15, 497529 (2002)

7. Gekhtman, M., Shapiro, M., Vainshtein, A.: Cluster algebras and Poisson geometry. Mosc. Math. J. 3, 899-934 (2003)

8. Goodearl, K.R., Yakimov, M.: Poisson structures of affine spaces and flag varieties. II. Trans. Am. Math. Soc. (2009, in press). Preprint math.QA/0509075

9. Kogan, M., Zelevinsky, A.: On symplectic leaves and integrable systems in standard complex semisimple Poisson-Lie groups. Int. Math. Res. Not. 32, 1685-1702 (2002)

10. Kostant, B., Wallach, N.: Gelfand-Zeitlin theory from the perspective of classical mechanics. I. In: Studies in Lie Theory. Progress in Mathematics, vol. 243, pp. 319-364. Birkhäuser, Boston (2006) 